This is an electronic reprint of the original article. This reprint may differ from the original in pagination and typographic detail.

Author(s): Laitinen, Mikko; Rossi, Mikko; Julin, Jaakko; Sajavaara, Timo

Title: $\quad$ Secondary electron flight times and tracks in the carbon foil time pick-up detector

Year: $\quad 2014$

Version:

Please cite the original version:

Laitinen, M., Rossi, M., Julin, J., \& Sajavaara, T. (2014). Secondary electron flight times and tracks in the carbon foil time pick-up detector. Nuclear Instruments and Methods in Physics Research, Section B: Beam Interactions with Materials and Atoms, 336(October), 55-62. https://doi.org/10.1016/j.nimb.2014.06.014

All material supplied via JYX is protected by copyright and other intellectual property rights, and duplication or sale of all or part of any of the repository collections is not permitted, except that material may be duplicated by you for your research use or educational purposes in electronic or print form. You must obtain permission for any other use. Electronic or print copies may not be offered, whether for sale or otherwise to anyone who is not an authorised user. 


\title{
Secondary electron flight times and tracks in the carbon foil time pick-up detector
}

\author{
Mikko Laitinen, Mikko Rossi, Jaakko Julin, Timo Sajavaara \\ Dept. of Physics, P.O.Box 35, 40014 University of Jyväskylä, Finland \\ Corresponding author: Mikko Laitinen \\ Telephone : +358400994836 \\ Fax: +35814617411 \\ E-mail: mikko.i.laitinen@jyu.fi
}

\begin{abstract}
:
Carbon foil time pick-up detectors used in the time-of-flight measurements of $\mathrm{MeV}$ energy ions have been studied in connection to time-of-flight-energy spectrometer used for heavy ion elastic recoil detection analysis. In experimental coincident TOF-E data characteristic halos are observed around light element isobars, and the origin of these halos were studied. The experimental data indicated that these halos originate from single electron events occurring before the electron multiplication in the microchannel plate. By means of electron trajectory simulations, this halo effect is explained to originate from single electron, emitted from the carbon foil, hitting the non-active area of the microchannel plate. This electron creates a secondary electron from the surface and which ends up to the microchannel plate pore, is multiplied and create now a detectable signal. Other general timing gate parameters such as wire-to-wire spacing of the grids, acceleration potential of the 1st grid and the mirror grid potential gradient were also studied in order to improve the detector performance.
\end{abstract}

\section{Keywords:}

timing gate, carbon foil time pick-up detector, Time-of-Flight, ToF-ERDA, spectrometer. 


\section{Introduction}

Modern time-of-flight elastic recoil detection (ToF-ERD) spectrometers often use two carbon foil time pick-up detectors [1--3], similar to the design by Busch et al. [4]. This type of timing detector has typically five basic components (see Fig. 1): 1) The carbon foil that emits the electrons due to ion passage, 2) the toblerone-part which accelerates the electrons from the carbon foil and is accompanied by transparent grid, or mesh structures providing field free central region, 3) electrostatic mirror to bend the path of the electrons by 90 degrees back to the field free toblerone-part, 4) microchannel plate (MCP) for electron multiplication and 5) the anode to collect the electrons. In addition to these, the decision of using the timing gate in forward or backward direction related to the incident ion needs to be made.

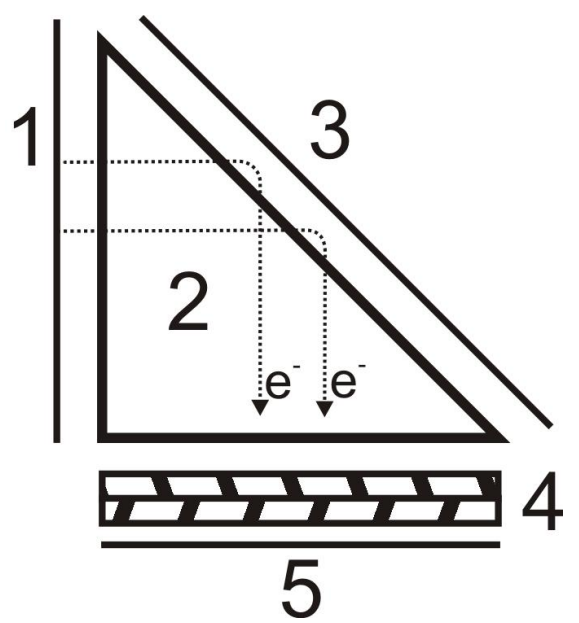

Figure 1. Basic components of the carbon foil time pick-up detector. This type of timing gate has five components: 1) carbon foil, 2) field free toblerone-part, 3) mirror grid, 4) MCP for electron multiplication and 5) anode to collect the electrons.

All of these individual detector components affect the timing performance through their material properties, structural geometry or by applied voltages. Energetic ion impact into the carbon foil will induce emission of zero to multiple secondary electrons, that can have wide energy and angular distributions [5,6]. The number of emitted electrons can be increased by other materials deposited on top of the carbon foil. These materials, such as $\mathrm{LiF}$ [2] or $\mathrm{Al}_{2} \mathrm{O}_{3}$ grown by atomic layer deposition (ALD) [7] can enhance the electron emission and therefore increase the detection efficiency for light ions. The emitted electrons having both the high energy and large emission angle perpendicular to the foil, can distort the timing signal already before the first accelerating grid causing nonisochronous electron transportation to the MCP. The grid spacing grid uniformity/smoothness and voltages applied to the mirror grid and toblerone-part also affect the electron trajectories before the MCP. The voltage, pore size and pore length of the MCP, and the distance between the individual MCP plates and their potential difference in chevron composition affect the rise time and width of the electron pulse [8]. Finally the anode design can have a big effect to the timing properties of the carbon foil time pick-up detectors in the time-of-flight measurements. In addition to these individual 
71 timing gate components, the decision of using the timing gate in forward direction related

72 to the incident beam i.e. the foil faces the beam first or in the backward geometry where

73 mirror faces towards the incident beam, needs to be made as well.

In this paper we focus on the timing pulse properties of the single carbon foil time pickup detector. Main emphasis is given to the individual parts before the MCP and how the different grid designs can affect the electron flight time properties from the carbon foil to the MCP. An explanation to the halo effect typically seen also in other studies $[9,10]$ around the hydrogen events in the ToF-E histograms is proposed. The MCP and anode part are left for less attention as ready MCP solutions with fast rise times (down to $300 \mathrm{ps}$ in standard products $[8,11]$ ) and matched anodes can be acquired commercially by several suppliers. For the case of timing gate orientation, one can for example win few centimeters in the ToF length if the first timing detector is facing forward and the second in backward electron emission direction. The forward direction can also produce more electrons due to the ion impact but their energy and especially their angular distribution is not that favorable than in the backward direction, according to the data in [6]. The angle and energy distributions of electrons and their effect to the timing properties are discussed in more detail in the following.

\section{Experimental and simulation parameters}

ToF-ERDA spectrometer with two carbon foil time pick-up detectors is located at the +15 degree beam line of the 1.7 MV Pelletron accelerator of the Accelerator Laboratory, University of Jyväskylä. ToF-ERDA method is best suited for light elements on heavy substrates, but the hydrogen recoils are often the most difficult ones to detect. One reason for this is the small stopping force of the detector carbon foils for hydrogen. Due to this, only a very small number of electrons is emitted from the carbon foil by the passing hydrogen ion. The single electron events are studied as a cause for the halos observed in the hydrogen isobars. The halos were experimentally studied with $2 \mathrm{MeV}{ }^{1} \mathrm{H}^{+}$beam scattered from a thick target. The model system in the simulations was the second timing gate.

\subsection{Timing gates of the ToF-ERDA spectrometer}

The first (T1) and second (T2) timing gates are not identical in our system. The main 


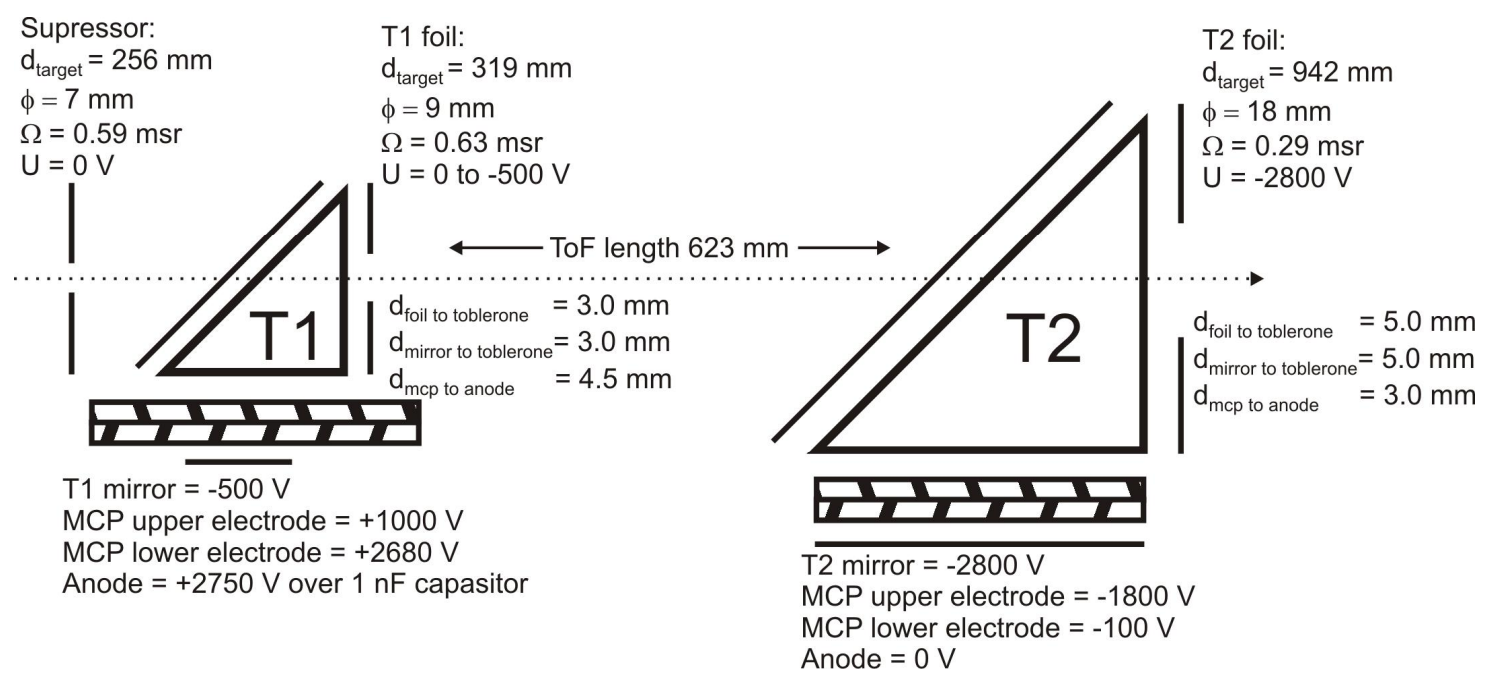

Figure 2. Distances, carbon foil sizes, solid angles and voltages of the Jyväskylä ToF-

111 The measures and voltages of the TOF telescope are shown in Fig. 2. The T2 has a solid angle of $0.29 \mathrm{msr}$, roughly half of the T1, although it is physically considerably larger. The total solid angle of the ToF-E telescope is governed by the T2 carbon foil holder (see also Fig. 3 b) as the silicon energy detector, placed right after the T2, has larger surface area of $450 \mathrm{~mm}^{2}$. The same MCP's (>40 mm active area, $12 \mu \mathrm{m}$ pore size, $\mathrm{d} / \mathrm{L}=1: 40$ ) are used in both of the timing gates. The anodes in both timing gates are modified from the original MCP stack-structure and are currently made from a printed circuit board. Anode

118 to MCP electrode distance is $4.5 \mathrm{~mm}$ and $3.0 \mathrm{~mm}$ for the $\mathrm{T} 1$ and $\mathrm{T} 2$, respectively. Supplier for the MCP's was Tectra [11].

The used voltages are different for T1 and T2. In the T1 the anode is at $+2750 \mathrm{~V}, \mathrm{MCP}$ lower (closer to anode) and upper electrodes are at $+2660 \mathrm{~V}$ and $+1000 \mathrm{~V}$ respectively, and the carbon foil can be grounded or slightly negatively biased. The T1 mirror grid needs to be negatively biased as it otherwise would accelerate free electrons towards the grid and the MCP; typically $-500 \mathrm{~V}$ is used in our measurements. The signal is taken from the $\mathrm{T} 1$ anode over a $1 \mathrm{nF}$ capacitor. For T2 $-2800 \mathrm{~V}$ is applied on both mirror and foil, $-1800 \mathrm{~V}$ on MCP upper electrode and on toblerone-part and anode is at ground potential.

High transparency grids in our timing gates compose of thin (diameters 25 and $20 \mu \mathrm{m}$ ) $\mathrm{Au}$ plated tungsten wires [12] that are point welded to their support frames (see Fig.3 a). The wire-to-wire spacing is $1.0 \mathrm{~mm}$ which was adopted from the timing gates developed earlier in our lab for nuclear physics experiments $[13,14]$. Distances from the foil to the first accelerating grid are $3.0 \mathrm{~mm}$ and $5.0 \mathrm{~mm}$ for $\mathrm{T} 1$ and $\mathrm{T} 2$, respectively. The distances of the mirror grids from the toblerone grids are the same than those of the foils. By using this type of point welded grid structure we have achieved better than $86 \%$ optical transmission through two timing gates (6 wire grids in total) together with the highly parallel and well aligned grid structure. 

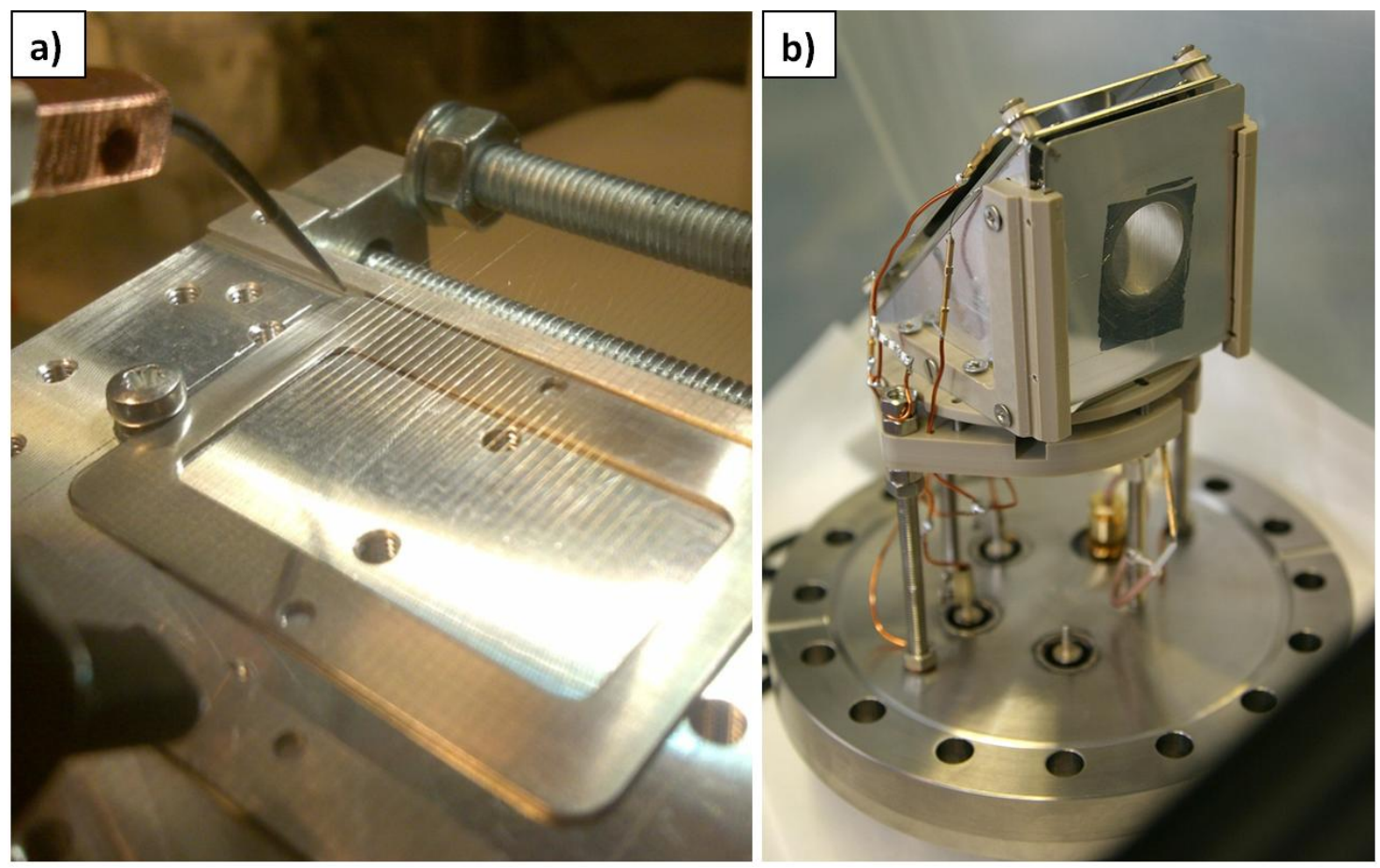

141 Figure 3. a) Point welding jig used in the fabrication of a grid. Wire (here $20 \mu \mathrm{m}$ ) is first wound around the jig where the pitch is determined by the pitch of the threads in both ends. Then, each wire is point welded from both ends to the frame holding the final grid structure. b) T2 timing detector fully assembled with $10 \mu \mathrm{g} / \mathrm{cm}^{2}$ carbon foil.

\subsection{Electron flight time and -path simulation at the timing gate}

A 2D model of the T2 timing gate was brought to the Simion program [15]. Simion is a software package primarily used for calculating electric fields and the charged particle trajectories in those fields [15].

Physically larger T2 was selected for the simulations as electron flight times, and possible time spreads were expected to be larger in it. Possible results were expected to scale down for the smaller T1. The model of the T2 timing gate had $20000 \times 20000$ pixels so that one pixel corresponded to about $3.5 \mu \mathrm{m}$. Thus $25 \mu \mathrm{m}$ wires in the real system had diameter of 7 pixels in the simulations.

The focus in the simulations was to find the optimal wire-to-wire spacing and potentials for the best achievable isochronous electron transport from the foil to the MCP electrode. The wire-to-wire spacing was varied from the ideal case (transparent, flat electric potential field) to $0.5 \mathrm{~mm}, 1.0 \mathrm{~mm}, 1.5 \mathrm{~mm}$ and $3.0 \mathrm{~mm}$. During the selection of the initial standard parameters for electron emission values, which was a sort of a compromise of the literature data available, mainly values from the Ref. [6] was finally used. The standard electron emission parameters were kept as: $\mathrm{E}_{\text {kin }}=4 \pm 4 \mathrm{eV}$ (Gaussian distribution) and incident angle of electron emission was uniformly distributed $\pm 30^{\circ}$ from the normal of the foil surface. The potentials were nominally the same as in the 
166 experimental configuration. Also other electron emission parameters from the carbon foil 167 were studied to cover wider electron energy and angular distributions and to verify their 168 effect to the electron flight times compared to the standard electron emission parameters.

170 In other simulations the electron scattering from the wire grids and from the MCP upper 171 electrode was studied in order to find the explanation to the longer than average electron 172 flight times seen in the experimental spectrum. The electron coming from the carbon foil 173 has energy about $1000 \mathrm{eV}$ when hitting the wires or the MCP electrode. A single 174 secondary electron was created from the single impact and the emission direction was set 175 as a specular reflection (incident angle $=$ reflection angle). Estimation of the created 176 secondary electron kinetic energy and the detection probability at the MCP pore was 177 problematic. Experimental data for the secondary and backscattering electron energies 178 and probabilities used in these simulations were taken from [16,17]. A function fitted to 179 the data shown in Fig. 4 a) was used by a random generator to determine the SE emission 180 energy after the initial impact. However, the data points in Fig. 4 a) were taken directly 181 from the Ref. [16] and are for the $550 \mathrm{eV}$ incident impact energy. Although the energy 182 data for incident electron reach only $550 \mathrm{eV}$ at [16] and not $1000 \mathrm{eV}$ it can be concluded 183 from [16] and [17] that most of the secondary electrons are always emitted with low 184 energies $<20 \mathrm{eV}$, having an intensity maximum at around $2 \mathrm{eV}$. This is regardless of the 185 impact energy. When going to higher energies the secondary electron emission intensity 186 drops close to zero for energies above $50 \mathrm{eV}$. However, there is always a small amount of 187 true backscattered/reflected electrons which have nearly the same energy as the incident 188 particle but their probability at 550 (and 1000) eV is very small. For this reason we have 189 neglected the material differences between [16] and our system $(\mathrm{Cu}$ at [16] vs. Au plated $190 \mathrm{~W}$ at wire grids and $\mathrm{Ni}$ at the MCP electrode material). 


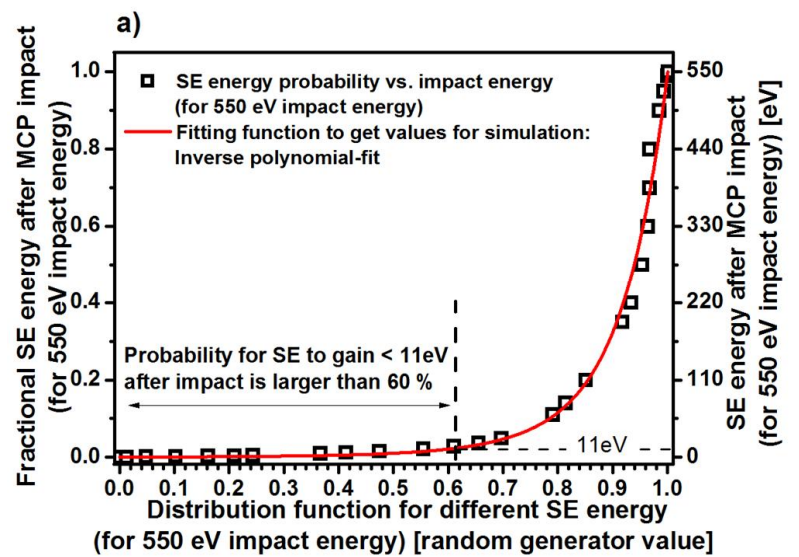

b)

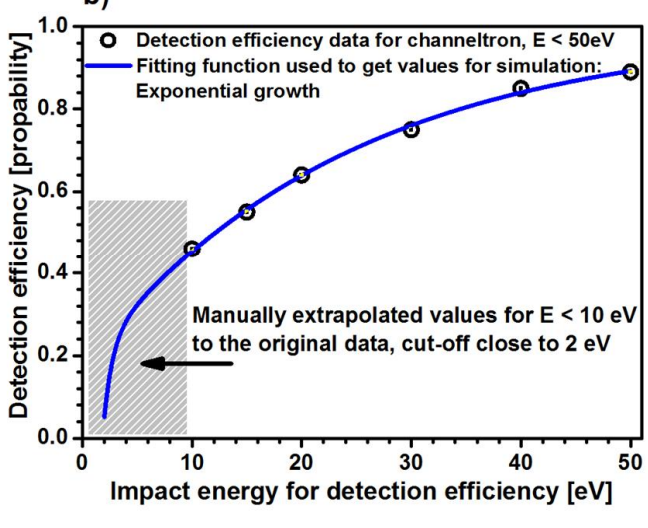

193 Figure 4. a) The secondary electron emission energy distribution as a distribution 194 function (probability) and b) MCP detection efficiency vs. electron impact energy. The probability function in a) was used to calculate the energy for the emitted electron after the primary electron had hit to the wire or the MCP surface. About $60 \%$ of these secondary electrons had less than $11 \mathrm{eV}(=0.02 \times 550 \mathrm{eV})$ energy although small amount of the emissions occurred almost at impact energies (true backscattering events). In b) estimated values for the detection efficiency of the MCP were taken from Ref. [49] down to $10 \mathrm{eV}$ and the points below the $10 \mathrm{eV}$ impact energy are approximated with a cut-off value of $<2 \mathrm{eV}$. Higher than $50 \mathrm{eV}$ impact energies were assumed to have constant detection efficiency ( 90\%) in the simulations.

203 The energy distribution of the secondary electrons (from the MCP electrode) influences 204 not only the electron flight time distribution before the MCP but also the detection 205 efficiency of the MCP. The detected hydrogen yield can be greatly affected by the MCP 206 detection efficiency for low energy electrons. The MCP detection efficiency for our configuration was not available for very low electron energies. An approximation for the MCP detection efficiency at smallest energies was obtained partly (down to $10 \mathrm{eV}$ ) from [18] and is shown in Fig. 4 b).

\section{Results}

\subsection{Electron paths and flight times: the effect of the grids and voltages}

Both wire-to-wire spacing and the applied foil, toblerone part and mirror grid voltage affect the electron transport from the foil to the MCP surface. Visual examples are presented in the Fig. 5. Here a) to c) represent different wire spacings (and also mirror grid distance change in c) which illustrate the need for obtaining both smooth acceleration from the foil to the 1st grid and a field free region inside the toblerone-part. The voltage configuration can affect the isochronous transportation of electrons and also the position information of the electron impact location on the MCP. This is demonstrated in Fig. 5 d) to f) where the time scale ( 200 ps ticks) is visualized by green markers. 


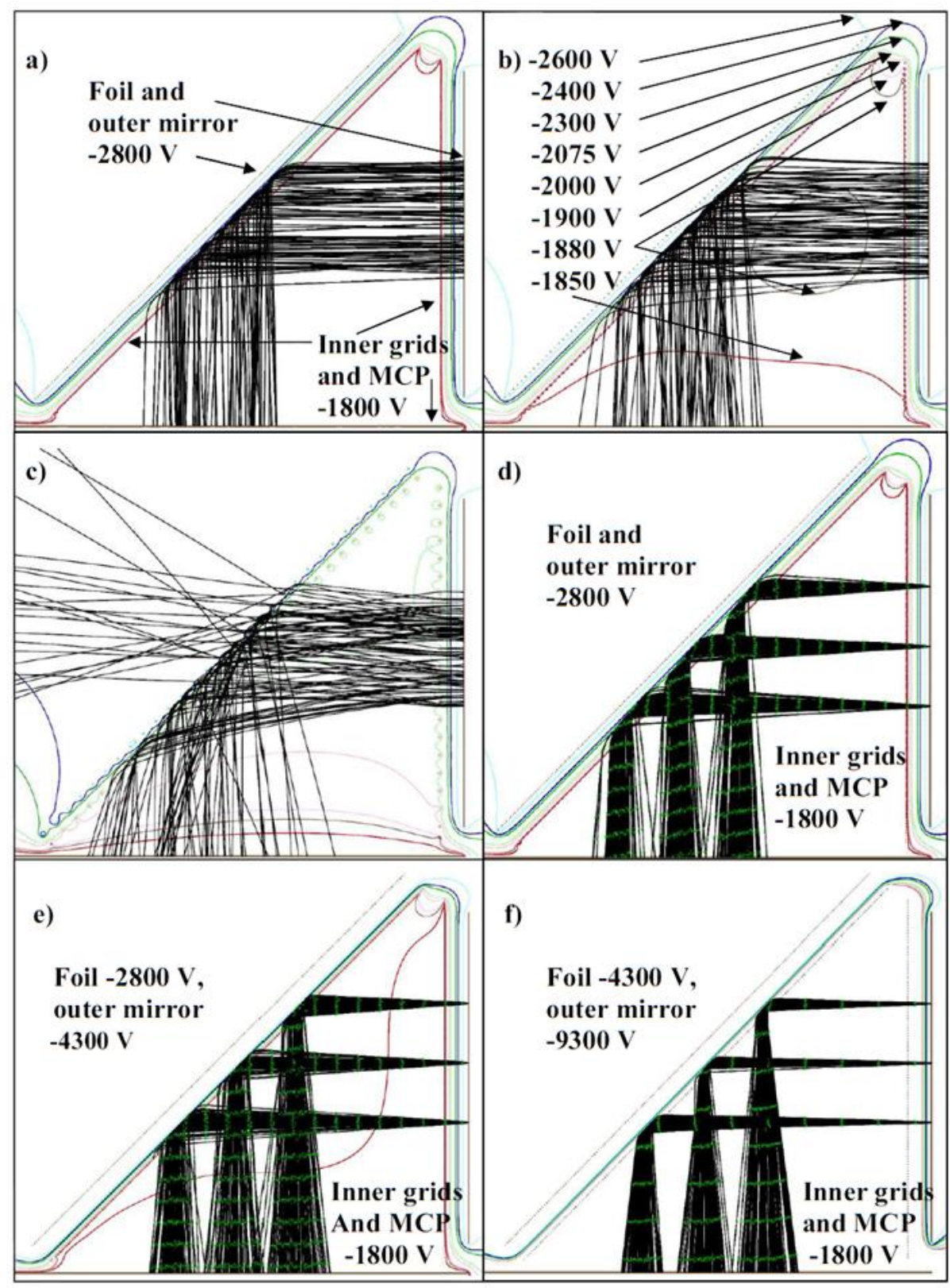

226 Figure 5. Electron trajectory simulations made with SIMION program for the T2 timing 227 gate with different wire-to-wire spacings and applied voltages. In $a$ ), $b$ ) and c) the wire228 to-wire spacings are $0.5 \mathrm{~mm}, 1 \mathrm{~mm}$ and $3 \mathrm{~mm}$, respectively, where b) represents the 229 experimental configuration in Jyväskylä. In c) the outer mirror grid is brought $1 / 3$ closer 230 to the inner mirror grid from the original perpendicular distance of $5.0 \mathrm{~mm}$. In d), e) and 231 f) the accelerating foil potential and the outer mirror potential has been changed for the $2320.5 \mathrm{~mm}$ wire-to-wire spacing -case. The (green) markers on the black electron paths 233 indicate $200 \mathrm{ps}$ time intervals. In these simulations, all electrons emitted from the foil 234 have initial energy of $(4 \pm 6) \mathrm{eV}$ with uniform distribution of \pm 40 degree perpendicular 235 to the foil surface (note: these emission values are slightly different than the standard 236 electron emission parameters used elsewhere in this paper). 
237 In general, the larger the individual wire-to-wire spacing is, the greater is the spreading of 238 the electron tracks. To reduce the electron track and electron flight time spread, one 239 should use small wire-to-wire spacing particularly in the 1st accelerating grid. To further 240 reduce the electron flight time spread, the potential difference between mirror grids 241 should be about 2 to 2.5 times higher than the potential difference from the foil to the $1 \mathrm{st}$ 242 accelerating grid (see Fig. 6 b). The high mirror grid potential reduces the time the 243 electrons spend in the mirror volume and thus reduces the deviation in the lengths of the 244 electron flight paths. This path length deviation is originally caused by the non-uniform 245 entering angle to the mirror volume. However, as seen from the Fig. 6 b), one cannot 246 increase the mirror voltage infinitely to reduce the flight time spread as when the 247 electrons are pushed too close to the toblerone wire grid their flight times start to deviate 248 from the shortest achievable value. This does not happen for the ideal grid because this 249 spread originates from the influence of the individual wire potentials of the non-ideal grid 250 to the electron flight paths.

252 One can reduce the electron flight time by increasing the acceleration potential and this 253 also reduces the electron flight time spread (Fig. 6 a). The spread almost saturates for 254 acceleration potentials above $1000 \mathrm{~V}$. Very high potential differences between the foil 255 and the toblerone can quickly lead to practical problems like bulging of the carbon foil 256 due to electric field or sparking, and it will clearly make the tandem effect [19] larger in 257 the case of T1, especially if the foil is in high potential and not grounded. 


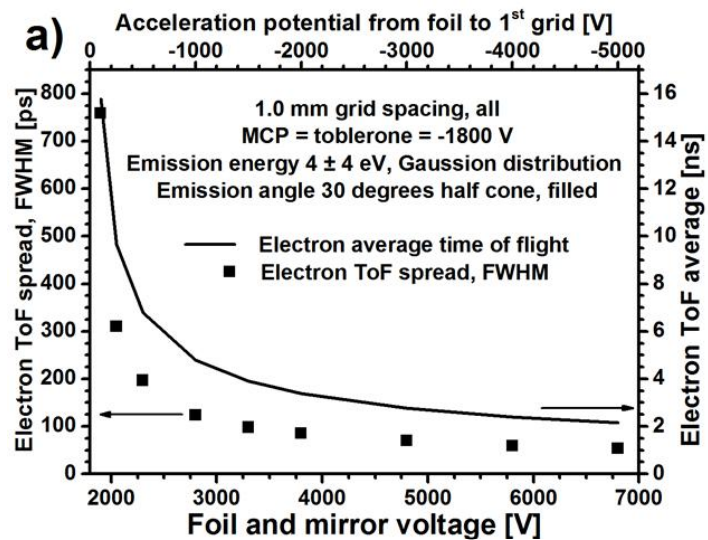

C) Toblerone and MCP $-1800 \mathrm{~V}$, foil and mirror $-2800 \mathrm{~V}$ C) Electron emission energy $4 \pm 4 \mathrm{eV}$, Gaussian distribution

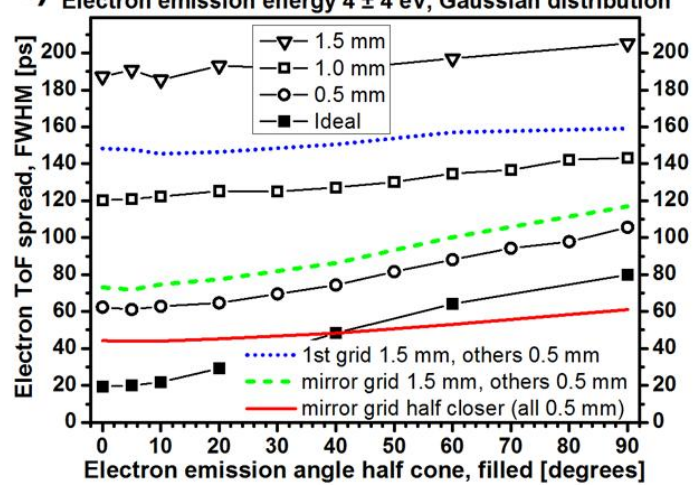

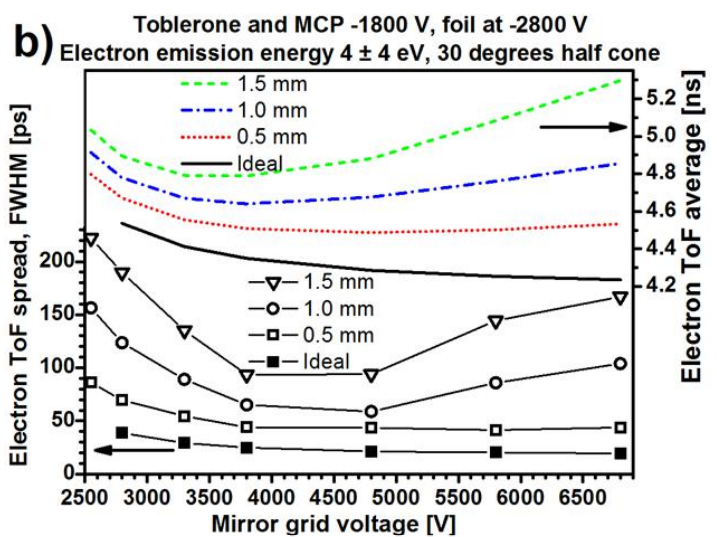

d) Toblerone and MCP $-1800 \mathrm{~V}$, foil and mirror $-2800 \mathrm{~V}$ d) Electron emission angle, half cone $=\mathbf{3 0}$ degrees, filled

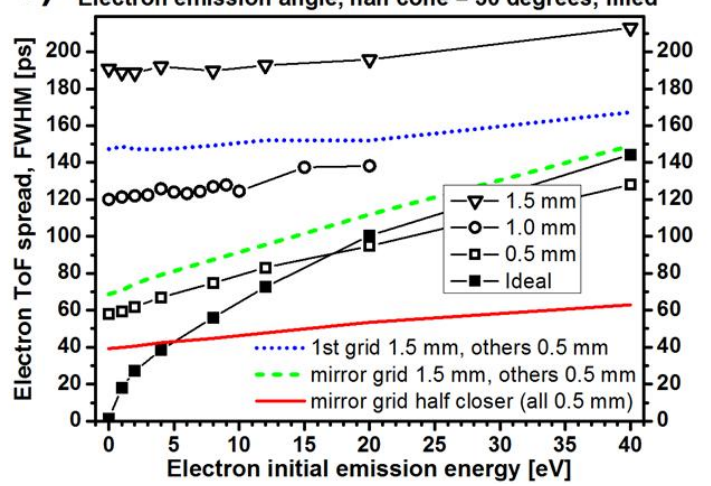

Figure 6. The effect of different timing gate and electron emission parameters to the electron flight time spread. a) The electron ToF spread and the electron flight times as a function of foil and mirror voltage. b) The TOF spread as a function of mirror grid potential for a fixed foil voltage. The TOF spread as a function of electron emission half cone c) and electron emission energy $d$ ).

Simulation results shown in the Fig. 6 c) and d) confirm that those electrons having the highest energy and widest emission angle when exiting the carbon foil will result the greatest time spread in electron flight times. While these events cannot be avoided, it is possible to minimize the timing spread caused by these electrons by design. Having as small wire-to-wire spacing as possible in the first acceleration grid, electric field strength in the mirror about two times as high as in the accelerating grid, one can still save some effort during the grid construction if having only $1.5 \mathrm{~mm}$ wire-to-wire spacing in the outer mirror grid. As our simulations indicate that, our current wire-to-wire spacing and used voltages in the timing gates are not optimal when electron flight time spread is concerned. By reducing the current $1.0 \mathrm{~mm}$ wire-to-wire spacing to $0.5 \mathrm{~mm}$ and by increasing the current mirror voltage from $-2800 \mathrm{~V}$ closer to $-4000 \mathrm{~V}$ for $\mathrm{T} 2$ one can reduce the estimated electron flight time spread from 125 ps to less than 50 ps (taken from Fig. 6 b)) without touching the foil or MCP/toblerone voltages.

The impact of the electron flight time spread in the single timing gate to the actually measured ion ToF spread in the two timing gates is not linear. It can be estimated that for 
the heavier ions, which emit more electrons when passing through the carbon foils, the spread of electrons flight times have smaller effect to the ion time-of-flight spread. This is due to more uniform electron emission cone at the carbon foil and because the fastest electrons will always cause the initial signal rise at the anode after the MCP. However, the lighter ions such as hydrogen to carbon which emit only one (even zero) to few electrons from the carbon foil, the single electron flight time is more important. This is because if the only emitted electron(s) flew the shortest and the longest flight times before the MCP in the T1 and T2, respectively, a larger spread for the ion ToF will be measured. If compared to the other effects $[3,20]$ causing the measured ion ToF spread, including the straggling in the first carbon foil, the overall contribution of the electron flight time spread in a single time gate is small. If for example timing resolution cannot be pushed down to $150 \mathrm{ps}$ regime (corresponding about $30 \mathrm{keV}$ for $4 \mathrm{MeV}$ ions for Jyväskylä ToF-ERDA [21]) and the kinematic effect due to large solid angle cannot be

\subsection{Halo around the hydrogen time-of-flight events}

Measured ToF-E isobars for the hydrogen have a clearly structured halo on both longer and shorter time-of-flights (see Fig. 7). For hydrogen these halo events can contribute at high energies up to $25 \%$ of all hydrogen events compared to the tight selection (see Fig.

304 7). Although this type of halo is most pronounced for the hydrogen, similar halos but 305 with reduced intensity can also be seen up to mass of carbon. The halo is typically about \pm 15 ns wide from the center of the as-expected ToF-value. By varying both the MCP and the foil (and mirror) voltages as shown in Fig. 7 it can be deduced that the events that give birth to the halo are generated after the foil and before the MCP. As seen in Fig. 7 b) the T2 foil voltage reduction (nominally $-1000 \mathrm{~V}$ compared to the MCP upper surface electrode) also spreads out the halo to the right side, longer ToF, but not completely smooths it out. The Fig. 7 c) and d) on the other hand indicate that the halo is generated from those events that have longer than normal $\mathrm{ToF}$ and especially from those events that have smaller than average pulse height as they vanish earlier with smaller MCP voltages (gain) compared to the main peak intensity. This pulse height difference in the halo and the main peak was also confirmed with the fast CAEN N6751 digitizer unit with full signal shape recording. From the Fig. 7 b) it is also more evident that there is a small gap between the halo-events and the main peak in which less events are detected. This shallow event free gap is more clearly visible in Fig. 9. 


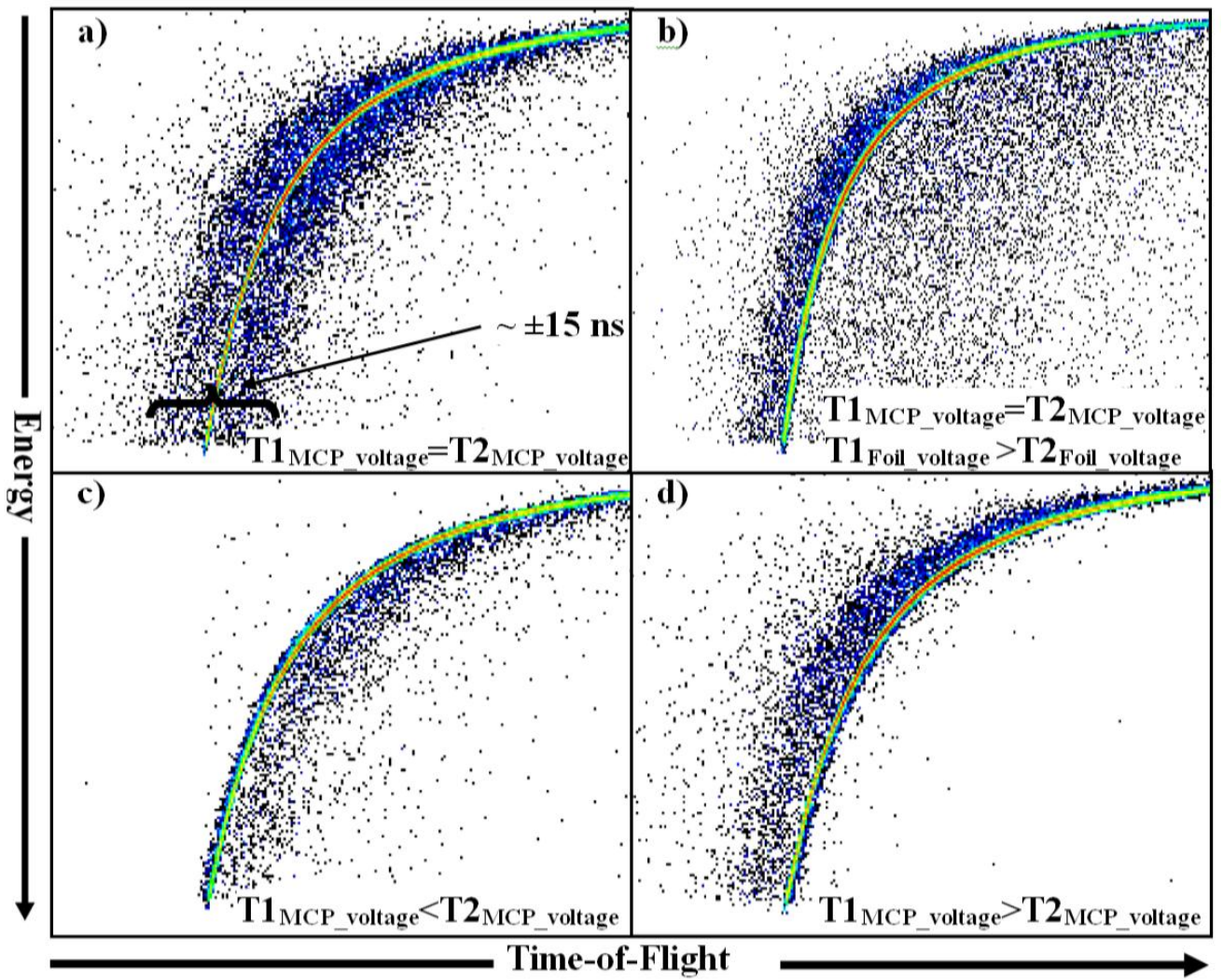

Time-of-Flight

321 Figure 7. Time-of-flight-energy histogram showing the influence of the MCP voltage 322 and foil voltage to the scattered hydrogen ion TOFs. When operating at nominal voltages 323 for our system, hydrogen has a clear background distributing over \pm 15 ns from the main 324 isobar as well as lighter background spreading further away as shown in a). In b) only 325 the $T 2$ foil has reduced voltage and therefore only smaller electron energies are 326 available within the T2. In c) only T1 MCP gain has been lowered so that only multiple 327 electron events generate detectable signals at T1 (similarly for T2 in d). 


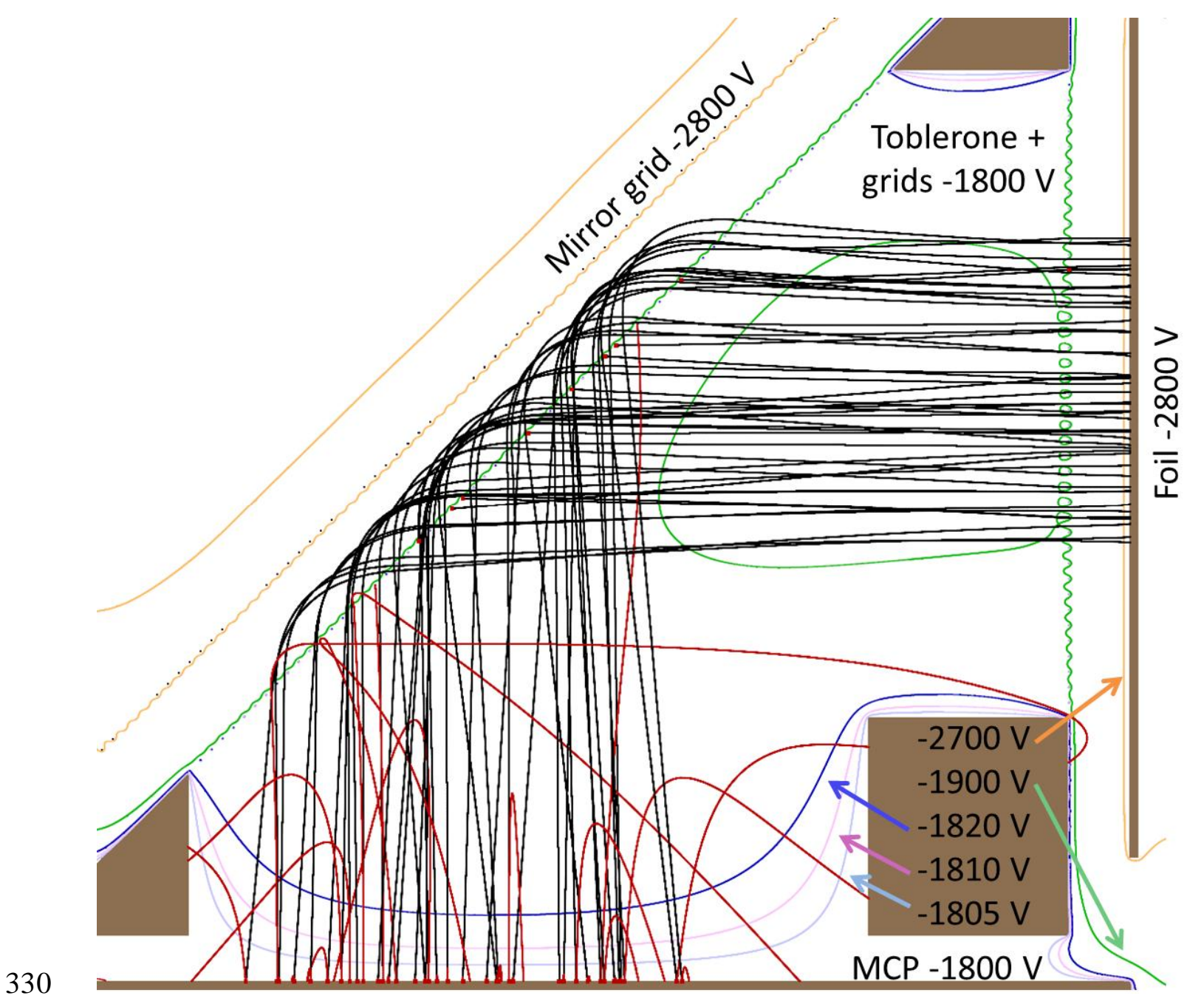

331 Figure 8. Electron paths (43) in our current T2 timing gate simulated with nominal 332 parameters. Single secondary electron emission was set to occur due to the primary 333 electron impact. About half of the secondary electrons (48 in total) from the MCP have 334 too little energy to be visible in this scale. In total, 10 secondary events end up to the 335 toblerone side mirror grid and 5 to the walls of the toblerone block, while majority of the 336 secondary electrons from the MCP surface end up back to the MCP surface and can 337 generate a signal.

338 Figure 8 shows simulated electron trajectories in a timing gate. In Fig. 8 most of the 339 secondary electron paths are not even visible and do not reach the $-1805 \mathrm{~V}$ equipotential 340 line above from the MCP as SE energy distribution intensity maximum is smaller than 5 $341 \mathrm{eV}$. For the same reason most of the SEs created by the primary electron hitting the wire 342 grids cannot escape the potential field of the individual wire. 


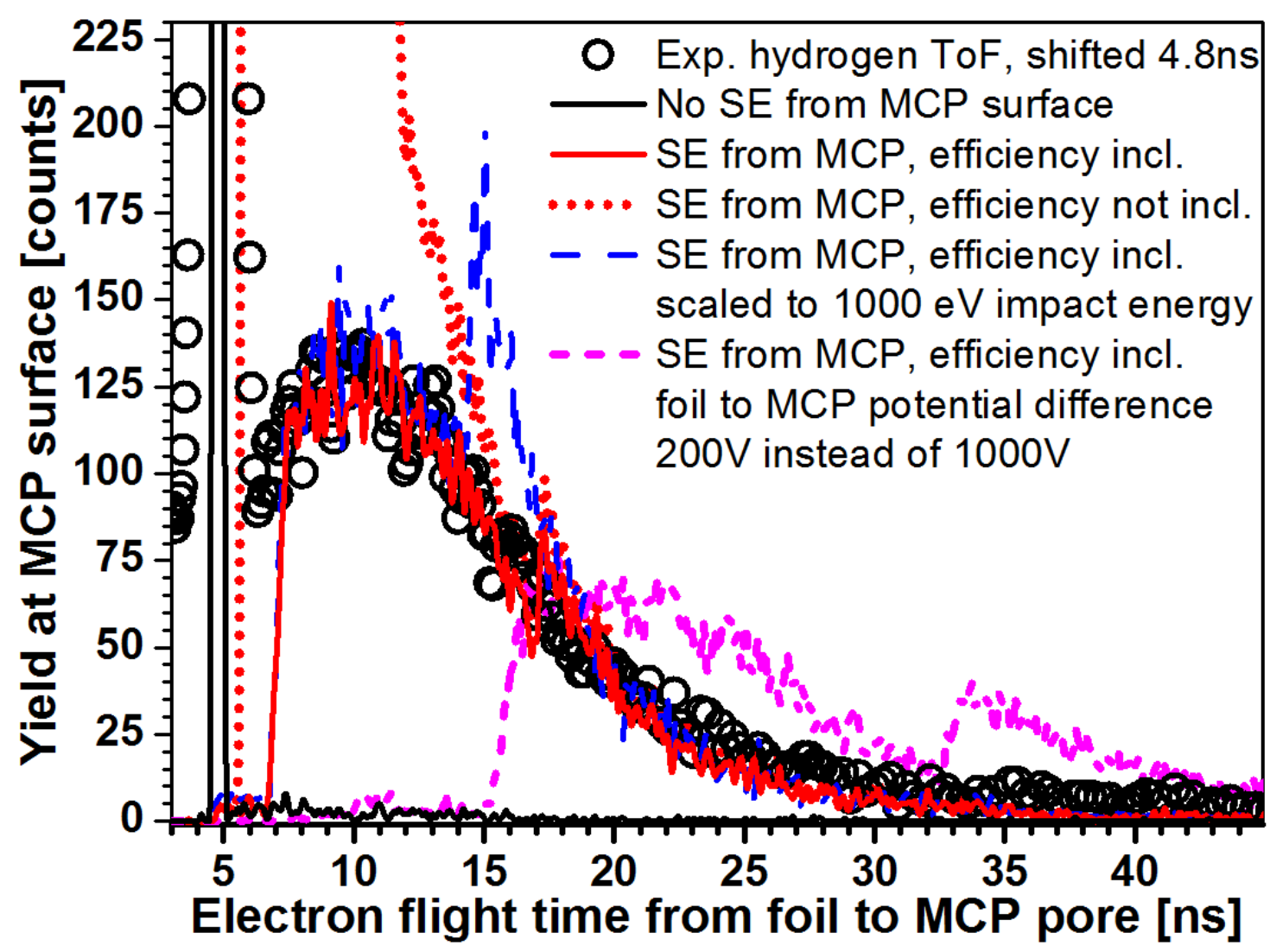

345 Figure 9. Experimental time-of-flight data from the hydrogen ions and corresponding 346 electron flight time simulations from the carbon foil to the MCP surface in our current T2 347 detector. The use of $1000 \mathrm{eV}$ impact energy instead of $550 \mathrm{eV}$ changes very little the 348 overall shape of the extended flight times with the exception of the shifted peak which is 349 present at the simulations (see text). The solid black line represents the case in which 350 scattering/secondary events only from the wire grids are considered. The dashed pink 351 line shows the extended electron flight times for reduced foil and mirror potentials. In the 352 experimental data the main peak (at $4.8 \mathrm{~ns}$ ) has about $75 \%$ of all events detected.

In the Fig. 9 are shown both the experimental data and simulated electron flight times in different cases. The experimental data in Fig. 9 is shifted $+4.8 \mathrm{~ns}$ which corresponds the simulated, as-expected, electron flight time from the foil to the MCP. As seen from the Fig. 9 only a very small contribution comes from the events that have scattered only from the wires and scattering from the MCP surface dominates. The case in wich MCP detection efficiency for different energies is not included (pointed line) the electron flight times between 6 and $15 \mathrm{~ns}$ are considerably pronounced compared to the experimental 360 data. However, if the MCP efficiency estimation for low electron energies is included, the 361 yield for the shortest secondary electron flight times between 6 and 15 ns drops 362 considerably, and simulations agree with the experimental data. There is, however, a 363 small peak in the simulation data at about $17 \mathrm{~ns}$ (and a larger peak at $\sim 15 \mathrm{~ns}$ for impact 364 energies scaled to $1000 \mathrm{eV}$ ). The origin of this extra peak, which changes very little the 365 overall shape of the extended flight times, is due to the highest energy electrons which 
have emitted from the MCP surface. The high energy electrons, most likely backscattered ones, have enough energy to wiggle back to the mirror and foil grids in the toblerone-part before ending back to the pore of the MCP. As this type of peak is not seen in the experimental data it can be concluded that the high energy backscattering yield is overestimated in the simulation parameters. If voltage of $200 \mathrm{~V}$ is used for both foil and mirror instead of $1000 \mathrm{~V}$, the simulated distribution of secondary electrons (Fig. 9) becomes much broader. The same effect is visible in Fig. $7 \mathrm{~b}$ ) where roughly the same parameters were in use in an experiment.

\section{Conclusions}

Electron transport properties in the carbon foil time pick-up detectors, being in use in the Jyväskylä ToF-ERD setup, have been investigated. Experimentally detected halo around the hydrogen isobar in the ToF-E histogram could be reproduced by simulations and its origin is understood. Experimentally it was confirmed that the halo events had smaller MCP pulses indicating that they originate from single electron events. In the simulations the halo was confirmed to form from those secondary electrons from the MCP surface that ended up to the active MCP pore after a short time period.

More simulations were made to study the different wire-to-wire spacings and voltages in the grids for electrons with different incident energy and angle. By decreasing the wireto-wire spacing and increasing the acceleration potential together with the mirror voltage, smaller flight time spreads were observed. By using less tightly spaced mirror grids (1.5 $\mathrm{mm}$ spacing), $0.5 \mathrm{~mm}$ spaced toblerone grids and 2-2.5 higher mirror potential difference than for the acceleration the timing spread reduces considerably.

\section{Acknowledgements}

This work was supported under the auspices of Finnish Centre of Excellence Programme 2006-2014 (Project No. 213503, 251353 Nuclear and Accelerator Based Physics), Finnish Funding Agency for Technology and Innovation (Tekes) project ALEBOND (decision no. 40079/08), MECHALD (decision no. 40207/11) and HIUDAKE (decision no. 70027/11) and Tekes EU-regional funds project (decision no. 70039/08).

\section{References}

[1] C. Kottler, M. Döbeli, F. Glaus, M. Suter, Nucl. Instr. and Meth. B, 248 (2006), p. 155.

[2] Z. Siketić, I.B. Radović, M. Jakšić, Nucl. Instr. and Meth. B, 266 (2008), p. 1328.

[3] S. Giangrandi, T. Sajavaara, B. Brijs, K. Arstila, A. Vantomme, W. Vandervorst, Nucl. Instr. and Meth. B, 266 (2008), p. 5144. 
408 [4] F. Busch, W. Pfeffer, B. Kohlmeyer, D. Schüll, F. Pühlhoffer, Nucl. Instr. and Meth., $409171(1980)$, p. 71.

410 [5] M. Burkhard, H. Rothard, C. Biedermann, J. Kemmler, K. Kroneberger, P. Koschar, 411 O. Heil, K. Groeneveld, Phys. Rev. Lett., 58 (1987), p. 1773.

412 [6] C.G. Drexler and R.D. DuBois, Phys. Rev. A, 53 (1996), p. 1630.

413 [7] M. Leskelä and M. Ritala, Thin Solid Films, 409 (2002), p. 138.

414 [8] Hamamatsu, MCP \& MCP assembly - selection guide, 415 http://www.sales.hamamatsu.com/resources/pdf/etd/MCPassy TMCP0001E09.pdf $416 \quad(25.2 .213)$.

417 [9] S. Giangrandi, Low-energy Elastic Recoil Detection and Ion Beam Analysis for 418 quantitative elemental profiling of thin films (KU Leuven, 2010) $\mathrm{PhD}$ thesis.

419 [10] T. Sajavaara, Heavy ion recoil spectroscopy of surface layers (University of 420 Helsinki, 2002) $\mathrm{PhD}$ thesis.

421 [11] Tectra, Microchannel Plates and Microchannel Plate Detectors, 422 http://www.tectra.de/MCP.htm (25.2.1013).

423 [12] Luma-metal, Gold Plated Tungsten Wire, http://www.luma-metall.se/products/gold424 plated-tungsten-wire (25.2.2013).

425 [13] W. Trzaska, V. Lyapin, V. Maslov, Nucl. Instr. and Meth. B, 197 (2002), p. 288.

426 [14] W. Trzaska, V. Lyapin, T. Alanko, M. Mutterer, J. Räisänen, G. Tjurin, M. Wojdyr, 427 Nucl. Instr. and Meth. B, 195 (2002), p. 147.

428 [15] Simion 8.1, SIMION® Ion and Electron Optics Simulator, Scientific Instrument 429 Services, Inc.

430 [16] V. Baglin, I. Collins, O. Gröbner, B. Henrist, N. Hilleret, and G. Vorlaufer, in Proc. 431 Int. Workshop on Two-Stream Instabilities in Particle Accelerators and Storage Rings, 432 KEK, (2001).

433 [17] B. Henrist, N. Hilleret, M. Jimenez, C. Scheuerlein, M. Taborelli, and G. Vorlaufer, 434 in proceedings of ECLOUD, Geneva, 15-18 April 2002 (CERN Report CERN-2002-001, 435 2002), p. 75.

436 [18] F. Bordoni, Nucl. Instr. and Meth., 97 (1971), p. 405.

437 [19] M. Döbeli, R. Ender, V. Liechtenstein, D. Vetterli, Nucl. Instr. and Meth. B, 142 438 (1998), p. 417. 
439 [20] S. Giangrandi, K. Arstila, B. Brijs, T. Sajavaara, A. Vantomme, W. Vandervorst, 440 Nucl. Instr. and Meth. B, 261 (2007), p. 512.

441 [21] Laitinen M., Rossi. M., Julin J., T. Sajavaara, Time-of-flight-energy spectrometer 442 for elemental depth profiling - Jyväskylä design, Submitted to Nucl.Instr.and 443 Meth.B(2013).

444 [22] Laitinen M. and T. Sajavaara, Trajectory bending and energy spreading of charged 445 ions in time-of-flight telescopes used for ion beam analysis, Accepted to Nucl.Instr.and 446 Meth.B(2014). 